\title{
Miniature probe for dual-modality photoacoustic microscopy and white-light microscopy for image guidance: A prototype toward an endoscope
}

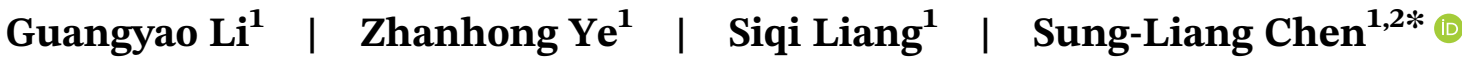

\begin{abstract}
${ }^{1}$ University of Michigan-Shanghai Jiao Tong University Joint Institute, Shanghai Jiao Tong University, Shanghai, China

${ }^{2}$ State Key Laboratory of Advanced Optical Communication Systems and Networks, Shanghai Jiao Tong University, Shanghai, China
\end{abstract}

\section{*Correspondence}

Sung-Liang Chen, University of MichiganShanghai Jiao Tong University Joint Institute, Shanghai Jiao Tong University, Shanghai 200240, China.

Email: sungliang.chen@sjtu.edu.cn

Funding information

National Natural Science Foundation of China, Grant/Award Number: 61775134

\begin{abstract}
In this study, a novel photoacoustic microscopy (PAM) probe integrating white-light microscopy (WLM) modality that provides guidance for PAM imaging and complementary information is implemented. One single core of an imaging fiber bundle is employed to deliver a pulsed laser for photoacoustic excitation for PAM mode, which provides high resolution with deep penetration. Meanwhile, for WLM mode,
\end{abstract}

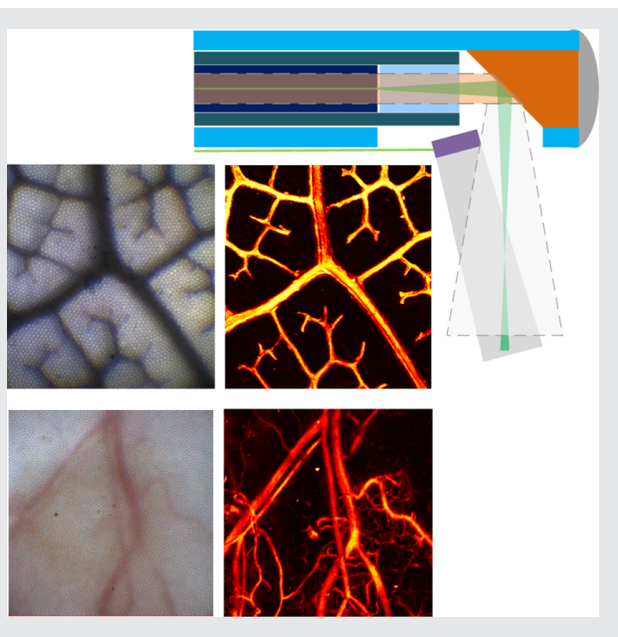
the imaging fiber bundle is used to transmit two-dimensional superficial images. Lateral resolution of $7.2 \mu \mathrm{m}$ for PAM is achieved. Since miniature components are used, the probe diameter is only $1.7 \mathrm{~mm}$. Imaging of phantom and animals in vivo is conducted to show the imaging capability of the probe. The probe has several advantages by introducing the WLM mode, such as being able to conveniently identify regions of interest and align the focus for PAM mode. The prototype of an endoscope shows potential to facilitate clinical photoacoustic endoscopic applications.

\section{K E Y W O R D S}

dual modality, image guidance, miniature probe, photoacoustic endoscopy, photoacoustic microscopy, white-light microscopy

\section{1 | INTRODUCTION}

Photoacoustic microscopy (PAM) is a novel noninvasive imaging technology that can reveal absorption contrast in biological tissue with high spatial resolution and provide structural and functional information through the spectroscopic imaging capability of PAM [1-3]. The high resolution is provided by either a focused laser beam

Abbreviations: 1D, one-dimensional; 2D, two-dimensional; ANSI, American National Standards Institute; AR-PAE, acoustic-resolution photoacoustic endoscopy; AR-PAM, acoustic-resolution photoacoustic microscopy; DOF, depth of focus; FOV, field of view; FWHM, full width at half maximum; GRIN, gradient index; LED, light-emitting diode; MAP, maximum amplitude projection; MMF, multimode optical fiber; OR-PAE, opticalresolution photoacoustic endoscopy; OR-PAM, optical-resolution photoacoustic microscopy; PAE, photoacoustic endoscopy; PAM, photoacoustic microscopy; PRF, pulse repetition frequency; SNR, signal-to-noise ratio; USAF, United States Air Force; WD, working distance; WLE, white-light endoscopy; WLM, white-light microscopy. 
or a focused acoustic transducer. The former is termed as optical-resolution PAM (OR-PAM) [4-15], and the latter acoustic-resolution PAM (AR-PAM) [16, 17]. Over the past few years, many attempts have been made to implement PAM as miniature probes, also called photoacoustic endoscopy (PAE) [18-35], which enables PAM of internal organs. PAE is desired for minimally invasive interventional procedures. Recently, many PAE applications have been demonstrated, such as gastrointestinal tract [18-20, 24-26, 29, 31, 33, 34], intravascular [22, 23, 28] and urogenital system imaging $[21,30]$. Similar to PAM, PAE can be categorized into OR-PAE $[21,23,25,26$, 29-32] and AR-PAE [18-20, 24], also depending on how resolution is determined. OR-PAE typically employs a single-mode optical fiber and a gradient-index (GRIN) lens to provide high resolution up to several micrometers despite sacrificing imaging depth. However, in clinical applications, scanning time could be prohibitively long because of more scanning pixels needed due to smaller step size for high-resolution imaging. Furthermore, the depth of focus (DOF) is much limited for high-resolution optical focusing, which leads to the difficulty in acquiring an in-focus image of internal organs because typically invisible during the endoscopic imaging process.

White-light endoscopy (WLE) is a widespread imaging technology, which utilizes a video camera to collect whitelight microscopy (WLM) images through an endoscope. [36-38] It is able to visualize lumen tissue of internal organs and allow online biopsy for effective clinical diagnostics [38]. WLE can also be integrated into other endoscopic imaging modalities. In this scheme, WLE can be used for image guidance during operation of other endoscopic imaging modalities as well as providing complementary information. For example, WLE was implemented within a $3.5-\mathrm{mm}$ optical coherence tomography probe for image guidance during imaging of human tympanic membranes [39, 40]. Besides, integrated WLE and fluorescence endoscopy was utilized to provide complementary information for tumor studies [41, 42]. High-resolution WLE and endoscopic ultrasound were used to evaluate early neoplasia in Barrett's esophagus [43]. Dual-modality PAE and WLE can be useful in biomedical applications. For example, WLE and PAE with transverse resolution of $\sim 37 \mu \mathrm{m}$ were utilized to provide complementary information in tumor diagnosis [26], yet the images were acquired by using two individual WLE and PAE endoscopes, respectively, which is inconvenient in clinical practice. PAE combined with WLE in a single probe has also been demonstrated [24, 33]. A catheter-based PAE probe was implemented and can fit within the $3.7-\mathrm{mm}$ diameter instrument channel of a commercial video endoscope [24]. However, the relatively low transverse resolution of $\sim 160 \mu \mathrm{m}$ may restrict clinical PAE applications in revealing fine structures. Another 9-mm probe utilizing a time reversal algorithm for tomographic PAE images achieved lateral resolution of $52 \mu \mathrm{m}$ [33], still the resolution is one order of magnitude lower than OR-PAM. Moreover, the probe size is relatively large. Therefore, it is of clinical importance to develop a probe that integrates WLE into PAE while high resolution and miniature probe size can be simultaneously achieved.

In this work, we present a novel design to combine PAM and WLM in a miniature probe. WLM allows image guidance for PAM and provides complementary information to PAM. The probe achieves high resolution for both PAM and WLM with miniature size of only $1.7 \mathrm{~mm}$ in diameter, demonstrating a promising a prototype for future development of an endoscope. High resolution of $7.2 \mu \mathrm{m}$ for PAM mode is achieved by using one single core of an imaging fiber bundle and a GRIN lens for light focusing. Meanwhile, high resolution of $31.3 \mu \mathrm{m}$ for WLM mode is obtained by sharing the same imaging fiber bundle and GRIN lens. The white-light source for WLM mode is delivered by an angle-cleaved multimode optical fiber (MMF) and is integrated into the probe. In part because of using some common components for PAM and WLM modes, the probe can be highly miniaturized. Further, lasers with two excitation wavelengths of 532 and $680 \mathrm{~nm}$ are coupled into one single core of the imaging fiber bundle to showcase scalable lateral resolution and imaging depth. In vivo imaging of mouse ear is conducted to show the imaging capability of the probe, including identifying regions of interest and checking the focus alignment for PAM mode. The additional WLM mode would be advantageous for improving the diagnostic efficiency to assist clinical PAM applications. Further development of an endoscope by integrating scanning capability to facilitate PAE applications while keeping high resolution and miniature size of the probe is also discussed.

\section{2 | METHODS}

\section{1 | Probe design and fabrication}

The schematic and photograph of the miniature dualmodality probe combining PAM and WLM are shown in Figure 1A,B, respectively. The probe employed fiber optic components for light and image delivery. An imaging fiber bundle (FIGH-10-500N; Fujikura, Japan) was employed to deliver pulsed laser for photoacoustic excitation in PAM mode and to transmit white-light images from the distal to the proximal end faces in WLM mode. The imaging fiber bundle, consisting of 10000 light guiding pixels, had an overall outer diameter of $600 \pm$ $20 \mu \mathrm{m}$ (image circle: $460 \mu \mathrm{m}$ ). The average diameter of 
the single cores was $\sim 3.0 \mu \mathrm{m}$ with a core-to-core spacing of $4.5 \mu \mathrm{m}$. The imaging fiber bundle was flexible, allowing a minimum bending radius of $25 \mathrm{~mm}$. Another MMF (M45L; Thorlabs) with core diameter of $400 \mu \mathrm{m}$ was used to deliver a white-light source for WLM mode. The distal end of the MMF was angle cleaved $\left(45^{\circ}\right)$ in order to realize side illumination, as shown in Figure 1. For WLM mode, a fixed spatial mapping existed between the two ends of individual cores, which means white-light images (from the backscattered light by tissue surfaces in our case) can be transmitted from one side to the other through the imaging fiber bundle. That is, the imaging fiber bundle can be utilized to "relay" images. On the other hand, for PAM mode, a single core of the imaging fiber bundle was used to deliver pulsed laser for photoacoustic excitation. To enable high resolution and long working distance (WD) for both PAM and WLM modes, a GRIN lens (GT-IFRL-060-005-50-C1; GRINTECH, Germany) with length of $1.4 \mathrm{~mm}$ and outer diameter of $600 \mu \mathrm{m}$ was adopted. The GRIN lens has antireflection coating on both end faces, which minimizes light reflection to $<0.5 \%$. As shown in Figure 1A, laser focusing for PAM mode and image transmission for WLM mode share
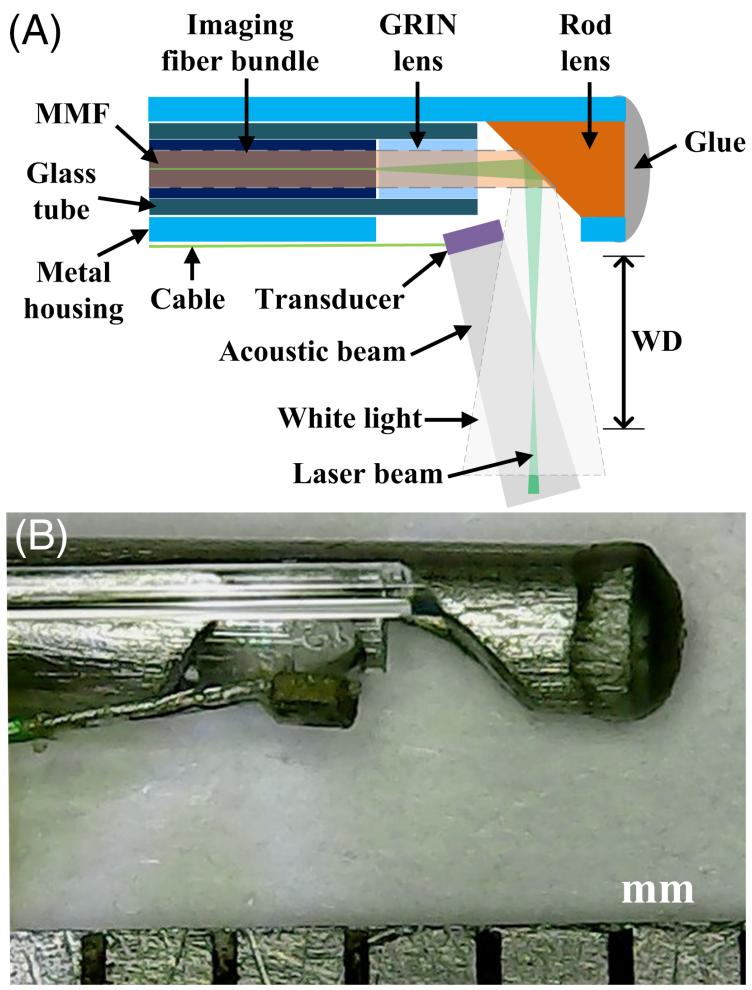

FI G URE 1 Schematic (A) and photograph (B) of the miniature probe combining photoacoustic microscopy (PAM) and white-light microscopy (WLM). Note that the multimode optical fiber (MMF) was attached along the outer wall of the metal housing the same optical components, the imaging fiber bundle and GRIN lens, and thus, both modes enjoy high resolution and long WD. The GRIN lens also contributes to wide field of view (FOV) for WLM mode. More explanations of long WD and wide FOV can be found in our previous work [32].

A custom-made thin-wall glass tube (inner diameter: $600 \mu \mathrm{m}$, outer diameter: $900 \mu \mathrm{m}$ ) was used to facilitate coaxial alignment and fixation of the imaging fiber bundle and the GRIN lens. To perform the side-view imaging, an aluminum-coated rod lens (47628, Edmund) with diameter of $1 \mathrm{~mm}$ and length of $2 \mathrm{~mm}$ was employed for $90^{\circ}$ light reflection for both PAM and WLM modes. Then, the glass tube and the rod lens were inserted into a custom-made metal housing (inner diameter: $1 \mathrm{~mm}$; outer diameter: $1.2 \mathrm{~mm}$ ), whose distal end was sealed with UV glue (NOA61; Thorlabs), as shown in Figure 1A. Note that a side window was opened on the sidewall of the metal housing for light transmission. A single-element ultrasonic transducer (AT23730; Blatek) with miniature dimensions $\left(0.6 \times 0.5 \times 0.2 \mathrm{~mm}^{3}\right)$ was used for acoustic detection. The transducer has central frequency of $\sim 40 \mathrm{MHz}$ with bandwidth of $\sim 60 \%$. The transducer was attached to the metal housing and slightly tilted to align the acoustic beam with the laser beam. With this arrangement, the imaging depth range is in part limited by the overlap of the two beams for laser excitation and acoustic detection. WD is decided from the transducer to the target (Figure 1A). Because of miniature components were used, the miniature size of the probe (1.7 $\mathrm{mm}$ in diameter) was achieved (Figure 1B). Note that the MMF was attached along the outer wall of the metal housing.

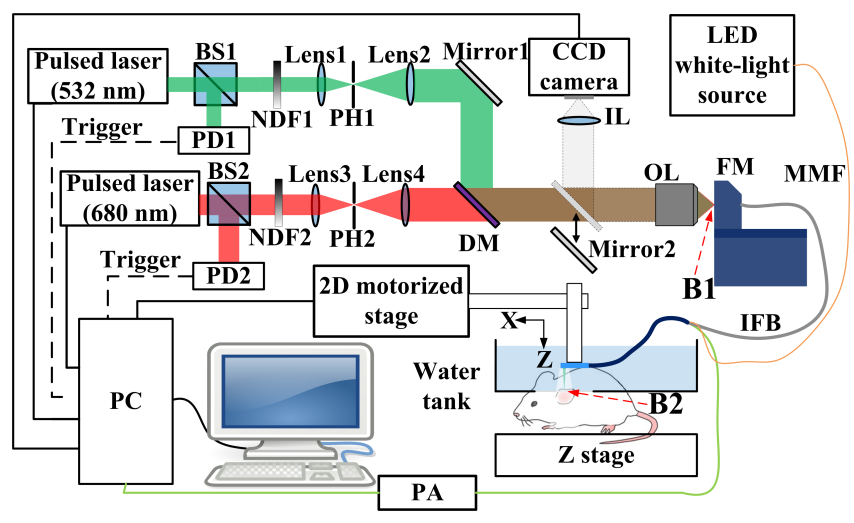

F I G URE 2 Schematic of the imaging system for performing photoacoustic microscopy (PAM) and white-light microscopy (WLM) modes. BS, beamsplitter; DM, dichroic mirror; FM, fiber mount; IFB, imaging fiber bundle; IL, imaging lens; NDF, neutraldensity filter; OL, objective lens; PA, preamplifier; PC, personal computer; PD, photodetector; $\mathrm{PH}$, pinhole 


\section{2 | Imaging system}

Figure 2 shows the setup of the imaging system for performing PAM and WLM modes. For PAM mode, we used a solid-state pulsed laser (FDSS532-Q3; CryLas, Germany) that emits laser at $532 \mathrm{~nm}$ with pulse repetition frequency (PRF) of $1 \mathrm{kHz}$, and another pulsed laser (Spitlight DPSS EVO I; InnoLas, Germany) that emits laser at $680 \mathrm{~nm}$ with PRF of $100 \mathrm{~Hz}$. Note that two wavelengths were used in this system, which is mainly to demonstrate the imaging capability of our probe in scalable lateral resolution and imaging depth. Further, it also manifests the feasibility of the probe for spectroscopic photoacoustic imaging, although not demonstrated directly. Using 10:90 beamsplitters, each of the 532 and $680 \mathrm{~nm}$ lasers were split into two laser beams. One laser beam with $10 \%$ power was used to provide the trigger for data acquisition, and the other with $90 \%$ power was attenuated, spatially filtered and expended. The respective $90 \%$ power of the 532 and $680 \mathrm{~nm}$ lasers was then combined by a dichroic mirror (DMLP605; Thorlabs). An objective lens (10×, numerical aperture: 0.3, BA310Met-T; Motic, China) was used to focus and couple the two lasers (both 532 and $680 \mathrm{~nm}$ ) into the imaging fiber bundle fixed on a fiber mount (HFF001; Thorlabs), which was further connected with a threedimensional motorized stage (not shown in Figure 2) to allow precise adjustment of the imaging fiber bundle. As mentioned previously, the laser for PAM mode was coupled into a single core by careful alignment of the proximal end face of the imaging fiber bundle (details described later). Two scanning schemes were employed in this work: linear scanning and rotary scanning. For linear scanning, the probe was mounted on a twodimensional (2D) motorized stage (M-L01.2A1; Physik Instrumente [PI], Germany) with its distal tip submerged into a water tank during image acquisition. The method is convenient to test the imaging capability of the probe. With the current system, the linear scanning time for an area of $1 \mathrm{~mm}^{2}(200 \times 200$ pixels with scanning step size of $5 \mu \mathrm{m}$ ) was less than $4 \mathrm{~min}$ by the $532 \mathrm{~nm}$ laser with PRF of $1 \mathrm{kHz}$. For rotary scanning, the probe was kept stationary while the sample was rotated by using a motor (not shown in Figure 2). This is to show the ability of the probe to acquire crosssectional images. The generated photoacoustic waves were detected by the transducer. Next, a preamplifier (CLC-10K0.5G-5510-S; Connphy) and a high-speed digitizer (CSE1422; Gage) were used for signal amplification and digital sampling at $200 \mathrm{MS} / \mathrm{s}$, respectively. Finally, a computer was used for image processing and display.

The laser beam focused by the objective lens and that by the GRIN lens are denoted as B1 and B2, respectively, as shown in Figure 2. By using the objective lens, the beam diameter of B1 of $\sim 2.2 \mu \mathrm{m}$ for the wavelength of $532 \mathrm{~nm}$ (and $\sim 2.8 \mu \mathrm{m}$ for $680 \mathrm{~nm}$ ) can be achieved, which is smaller than the average diameter of the single cores $(\sim 3.0 \mu \mathrm{m})$ and thus facilitates the laser coupling into a single core. To achieve this in experiment, careful alignment in two steps was conducted. First, the imaging fiber bundle was adjusted in the axial direction until a minimum spot size of B2 was observed, which indicates that the proximal end face of the imaging fiber bundle was aligned to the focal plane of B1 although B1 may be coupled into more-than-one cores. Second, the imaging fiber bundle was adjusted in the lateral direction until maximum power of B2 (ie, maximum coupling efficiency) was measured by a power sensor (S130VC; Thorlabs), which implies that B1 was coupled into a single core. The magnification from the focal plane of B1 to that of B2 is estimated to be $\sim 6$ by Zemax simulation [32]. Therefore, if B1 is coupled into an adjacent core, B2 will be shifted by $\sim 27 \mu \mathrm{m}[=4.5 \times 6]$. On the other hand, the acoustic detection range of the transducer at the focal plane of B2 can be calculated to be $\sim 0.4$ to $0.52 \mathrm{~mm}$ considering the full detection angle of $\sim 8.2^{\circ}$ to $10.5^{\circ}$ of the transducer (Figure S1C), which was measured using a similar approach based on a photoacoustic source [44]. Since the acoustic detection range is much larger than the shift of B2 due to coupling into an adjacent core, it is not required to couple $\mathrm{B} 1$ into a specific core. In other words, once B2 is within the acoustic detection range of the transducer (also see Figure 1A), the alignment of the laser and acoustic beams is achieved. Then, the transducer can be fixed with the metal housing for imaging experiment.

For WLM mode, a fiber-coupled light-emitting diode (LED) (MCWHF2; Thorlabs) was used to provide the white-light source. The LED was able to provide whitelight power of $27 \mathrm{~mW}$ at the MMF's output, which was used to illuminate phantoms or tissue. Then, as mentioned previously, the backscattered light was collected and transmitted by the GRIN lens and the imaging fiber bundle to the proximal end face. The same objective lens was to collimate the white-light image emitting from the imaging fiber bundle. For WLM mode, a mirror (Mirror2 in Figure 2) was inserted at a position to reflect the collimated white-light image. Note that Mirror2 was used to facilitate the switch between PAM and WLM modes (ie, Mirror2 inserted for WLM mode and moved away for PAM mode). Finally, an imaging lens was used to focus the white-light image onto a CCD camera (QHY5L-II; QHYCCD, China) to capture the WLM image. In practical applications, WLM image may be captured first (quicker), and PAM scanning can be conducted later (slower). 


\section{3 | RESULTS}

\section{1 | Resolution and imaging depth}

To measure resolution of the PAM and WLM modes, several experiments were conducted. For PAM mode, $6-\mu \mathrm{m}$ carbon fibers and $25-\mu \mathrm{m}$ tungsten wires placed at different depths at WD from $\sim 2.8 \mathrm{~mm}$ (corresponding to the focal length of $\sim 4.3 \mathrm{~mm}$ ) to $\sim 5.8 \mathrm{~mm}$ were imaged. The probe was scanned along the direction perpendicular to the carbon fiber (or the tungsten wire) by using the linear scanning scheme. Then, the one-dimensional (1D) profile was fitted by a Gaussian function, and lateral resolution can be determined by checking the full width at half maximum (FWHM) of the Gaussian fit. Figure 3A,B shows the XZ (also see Figure 2) photoacoustic B-mode images acquired at laser wavelengths of 532 and $680 \mathrm{~nm}$, respectively. Note that $Z=0$ corresponds to the focus (ie, $\mathrm{WD}=\sim 2.8 \mathrm{~mm}$ ). Also, $25-\mu \mathrm{m}$ tungsten wires were used for $Z=\sim 1.5$ to $3 \mathrm{~mm}$ at the excitation wavelength of $680 \mathrm{~nm}$, while $6-\mu \mathrm{m}$ carbon fibers were used otherwise. This is because the laser fluence of the $680 \mathrm{~nm}$ laser at deep depth was too low, and $25-\mu \mathrm{m}$ tungsten wires were used to provide stronger photoacoustic signals. Otherwise, the images will have too low signal-to-noise ratios (SNRs) to be visible. Figure 3C,D shows representative 1D profiles and Gaussian fits at $Z=0 \mathrm{~mm}$ for 532 and $680 \mathrm{~nm}$ lasers, respectively. Therefore, lateral resolution can be determined to be $7.2 \mu \mathrm{m}(532 \mathrm{~nm})$ and $12.2 \mu \mathrm{m}$ $(680 \mathrm{~nm})$. The curve for lateral resolution vs $\mathrm{Z}$ (ie, depth) is plotted in Figure 3E. As can be seen, the $532 \mathrm{~nm}$ laser enables higher resolution at superficial depth (eg, $Z=0 \mathrm{~mm}$ ), while $680 \mathrm{~nm}$ laser renders deeper penetration by maintaining moderate resolution (eg, $Z=2 \mathrm{~mm}$ ). The results suggest that our probe was able for scalable lateral resolution and imaging depth. Note that the measurement was conducted in water with negligible scattering. When the probe is used to image tissue, which has
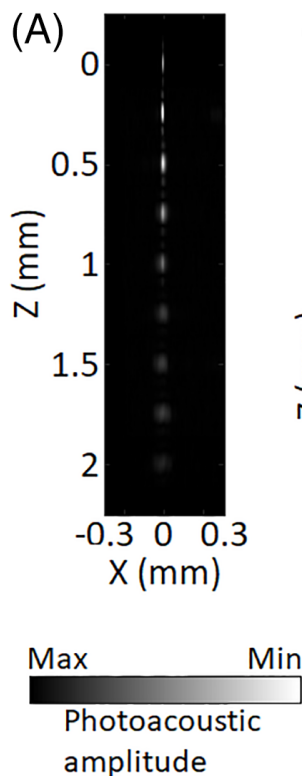

(B)
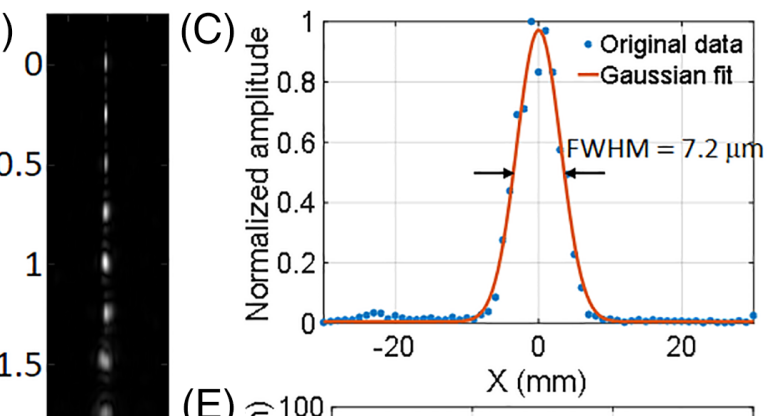

(E)

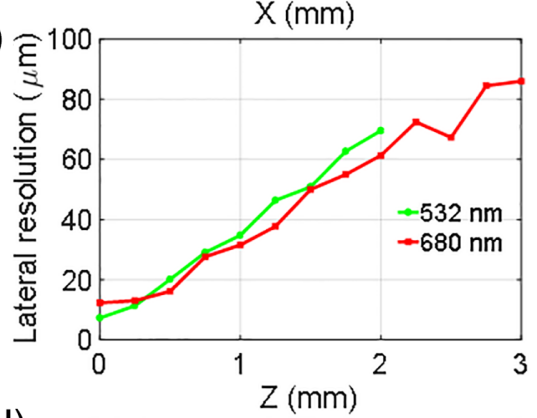

(G)

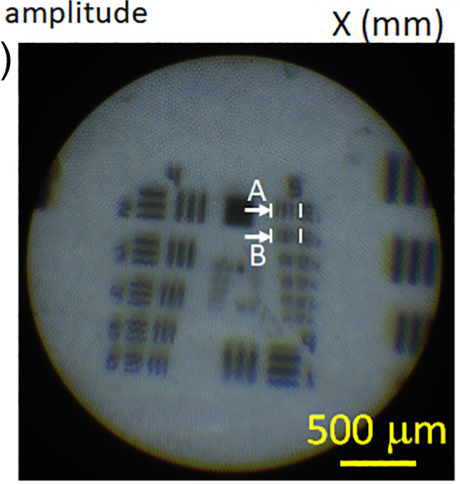

(H)

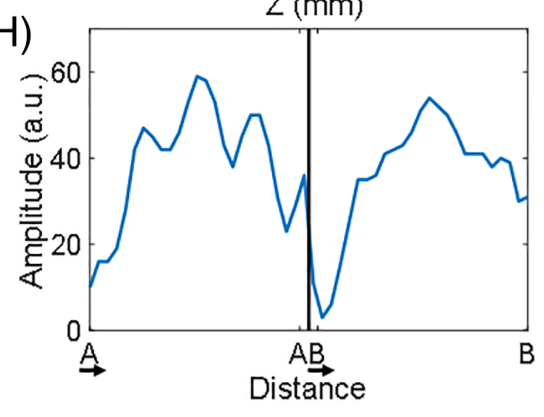

(D)

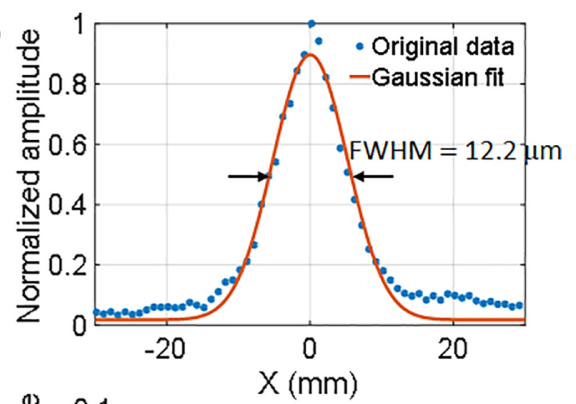

(F)

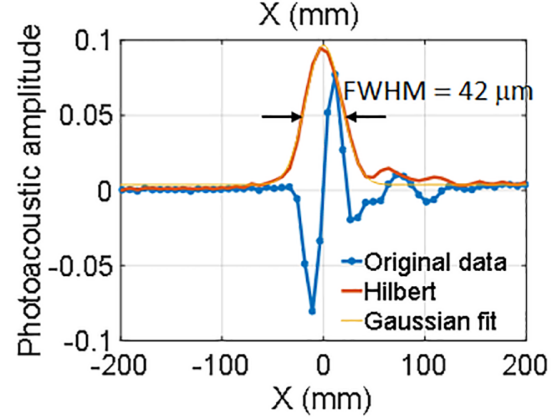

(I)

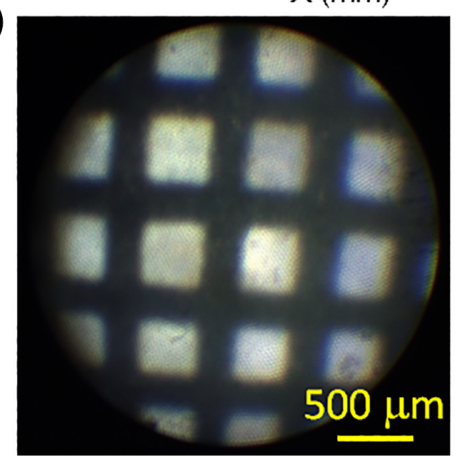

F I G U RE 3 XZ photoacoustic B-mode images acquired at laser wavelengths of $532 \mathrm{~nm}$ (A) and $680 \mathrm{~nm}$ (B). Note that $Z=0$ corresponds to the focus (ie, WD $=\sim 2.8 \mathrm{~mm}$ ). Representative $1 \mathrm{D}$ profiles and Gaussian fits at $Z=0 \mathrm{~mm}$ for $532 \mathrm{~nm}$ (C) and $680 \mathrm{~nm}$ (D) lasers. E, Lateral resolution vs $Z$ curve. F, Axial resolution measurement for photoacoustic microscopy (PAM) mode. G, The white-light microscopy (WLM) image of the USAF resolution test target. H, 1D profiles along the arrows indicated in (G). I, FOV measurement for WLM mode 
strong scattering at visible wavelengths (especially shorter wavelengths), lateral resolution of the $532 \mathrm{~nm}$ laser is expected to quickly degrade with increased depth, while that of the $680 \mathrm{~nm}$ (or longer wavelength) laser would be deteriorated gradually because of relatively weak scattering. Axial resolution is determined by the acoustic bandwidth of the transducer. The $6-\mu \mathrm{m}$ carbon fiber was imaged. The photoacoustic A-line profile and its Hilbert transform (envelope detection) are plotted in Figure 3F). By checking the FWHM of the envelope, the axial resolution was measured to be $\sim 42 \mu \mathrm{m}$.

Next, we characterized the resolution and FOV for WLM mode. A United States Air Force (USAF) resolution test target (R1DS1P; Thorlabs) was imaged by WLM mode using the probe, as shown in Figure 3G. Two 1D profiles along the arrows indicated in Figure $3 \mathrm{G}$ are plotted in Figure $3 \mathrm{H}$. As can be seen, the smallest resolvable bar spacing is $31.3 \mu \mathrm{m}$ (group 5, element 1). Thus, the resolution is better than $31.3 \mu \mathrm{m}$. Moreover, the FOV of WLM mode was estimated by imaging a mesh grid printed on a transparency. The linewidth and period of the mesh grid were 250 and $680 \mu \mathrm{m}$, respectively. The WLM image is shown in Figure 3I, and the FOV of $\sim 2.7 \mathrm{~mm}$ in diameter was achieved. Compared with PAM, the resolution of WLM is worse, which is in part limited by undersampling due to the intercore spacing. Computational approaches to restore pixilation-limited images could be used to enhance the resolution of WLM $[45,46]$.

To measure the imaging depth of our probe at 532 and $680 \mathrm{~nm}$ lasers, a needle with diameter of $530 \mu \mathrm{m}$ obliquely inserted into chicken breast was imaged. Then, $6-\mu \mathrm{m}$ carbon fibers were placed on the surface of the chicken breast, which was used as a reference. For fair comparison, the imaging depth was measured under similar laser fluence for the two laser wavelengths. Considering that the lateral resolution using $680 \mathrm{~nm}$ laser is worse than that using $532 \mathrm{~nm}$ laser, the pulsed laser energy used in this measurement was $\sim 305$ and $\sim 750 \mathrm{~nJ}$ for

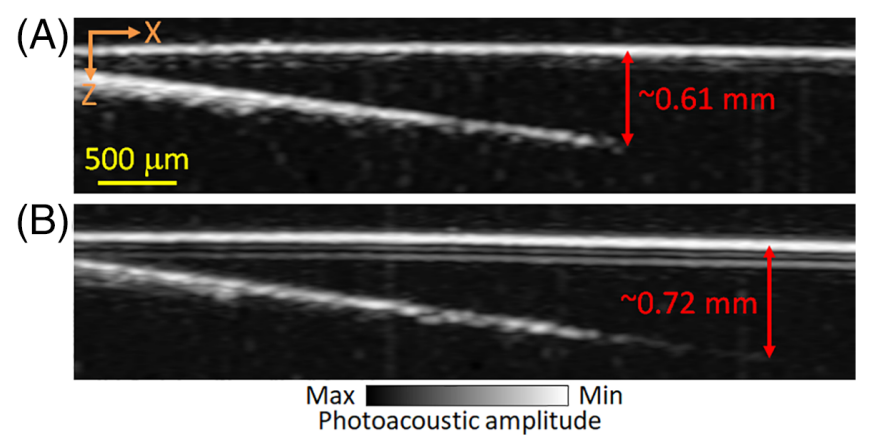

F I G U RE 4 XZ maximum amplitude projection (MAP) photoacoustic images of the needle obliquely inserted into chicken breast acquired at laser wavelengths of $532 \mathrm{~nm}$ (A) and $680 \mathrm{~nm}$ (B)
532 and $680 \mathrm{~nm}$ lasers, respectively. Figure 4 shows the acquired XZ maximum amplitude projection (MAP) photoacoustic images, where 16 times signal averaging was applied. Note that signal averaging was also used in measuring the maximum imaging depth in previous OR-PAM work [7]. As can be seen, our probe can clearly visualize the needle down to 0.61 and $0.72 \mathrm{~mm}$ (determined by SNRs $>20 \mathrm{~dB}$ ) beneath the reference surface by using 532 and $680 \mathrm{~nm}$ lasers, respectively. Thus, the imaging depth was determined to be $0.61 \mathrm{~mm}(532 \mathrm{~nm})$ and $0.72 \mathrm{~mm}(680 \mathrm{~nm})$. That is, the imaging depth using the $680 \mathrm{~nm}$ laser was improved by $\sim 18 \%$ compared with using the $532 \mathrm{~nm}$ laser. The penetration may be further enhanced by using a longer excitation wavelength such as $1064 \mathrm{~nm}[11,17]$.

\section{2 | Phantom imaging}

As mentioned previously, we conducted rotary scanning to show the feasibility to acquire cross-sectional images. Six black bristles (diameter: $\sim 210 \mu \mathrm{m}$ ) were imaged. They were placed around and roughly in parallel with the probe. The bristles had angle separation of $\sim 60^{\circ}$ and were at radial distance of $\sim 4.1$ to $6.5 \mathrm{~mm}$. During image acquisition, the sample was rotated with angular step size of $0.225^{\circ}$. Both WLM and PAM modes were performed in water. We first used WLM mode to align the probe's focus with one of the bristles, as shown in Figure 5A. Then, PAM mode was used to acquire the cross-sectional image, as shown in Figure 5B. The results also showcase the advantage of WLM mode in assisting PAM mode for the focus alignment, which shows potential to improve the efficiency in clinical endoscopic applications in future, especially for high-resolution PAE suffering from limited DOF.

To further demonstrate the quick focus alignment of PAM enabled by WLM mode, we imaged another sample of leaf skeletons dyed with black ink. The sample was covered by a thin layer of silicone to protect the ink from leaking out of the leaf skeleton. As mentioned previously, linear scanning was performed for convenience. WLM mode was acquired first when the probe was placed at a certain height (ie, a certain $\mathrm{Z}$ position) from the sample, as shown in Figure 6A. As can be seen, the WLM image is blurry. Then, PAM mode was conducted by scanning the probe over the XY plane, and the XY MAP photoacoustic image is shown in Figure 6B. As expected, the PAM image is blurry as well. Next, the probe was conveniently aligned with the sample through WLM mode, as shown in Figure $6 \mathrm{C}$, by adjusting the $\mathrm{Z}$ position of the probe $\sim 1 \mathrm{~mm}$ away from the previous $\mathrm{Z}$ position. Because the sample is located at the focal plane in Figure 6C, the FOV is narrower compared with Figure 6A. Similarly, 

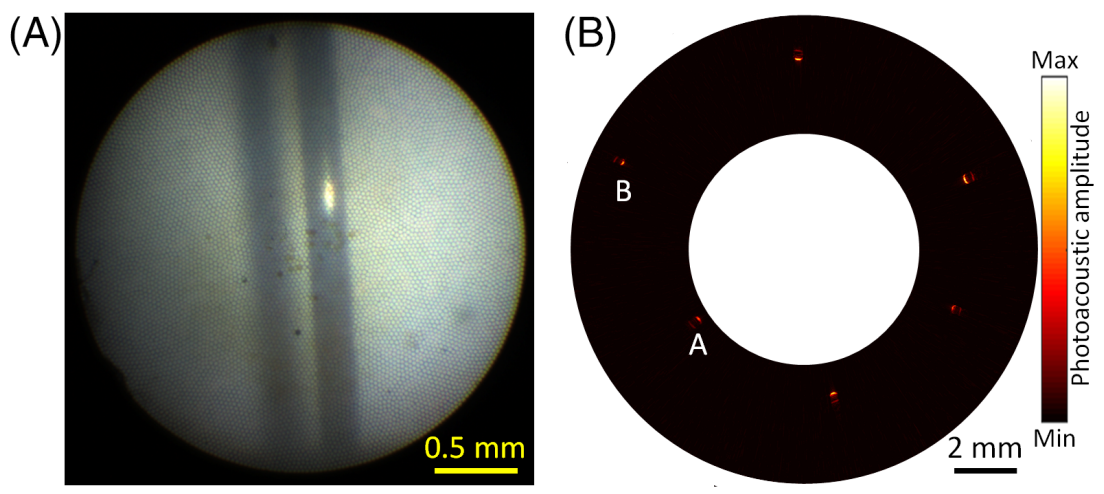

F I G U R E 5 White-light microscopy (WLM) image (A) and cross-sectional photoacoustic microscopy (PAM) image (B) of black bristles. Note that in (A), only one representative bristle was imaged. The left blurred bristle was a mirror image of the right clear one due to light reflection by the water tank used in this experiment. In (B), the six bristles were placed from small to large radial distance, where the smallest radial distance is $\sim 4.1 \mathrm{~mm}$ at position $\mathrm{A}$, and the largest is $\sim 6.5 \mathrm{~mm}$ at position $\mathrm{B}$

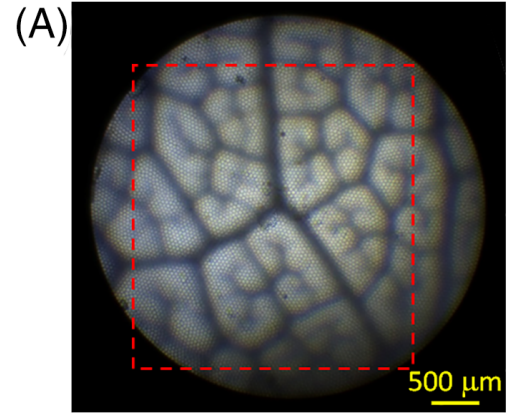

(B)

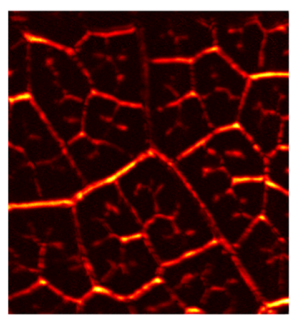

(C)

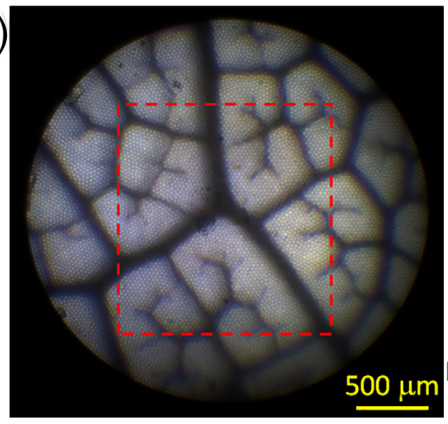

(D)

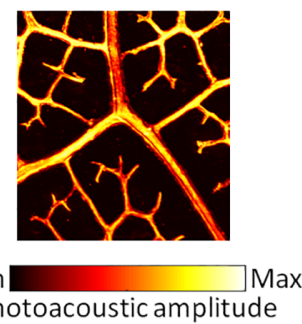

F I G U R E 6 White-light microscopy (WLM) image (A) and XY maximum amplitude projection (MAP) photoacoustic image (B) for the out-of-focus case. WLM image (C) and XY MAP photoacoustic image (D) for the in-focus case. A,B, The same scale bar in (A). $\mathrm{C}, \mathrm{D}$, The same scale bar in (C)

PAM mode was conducted by the probe at the new $\mathrm{Z}$ position, and the XY MAP photoacoustic image is shown in Figure 6D, which shows much clearer leaf skeletons. The results further verify the advantage of the additional WLM mode in the quick focus alignment for PAM. For the two pairs of dual-modality WLM and PAM images (ie, Figure 6A,B; Figure 6C,D), the dashed box in the WLM image corresponds to the PAM image for each pair. As expected, in both pairs, the leaf patterns in WLM and PAM images show high resemblance.

\subsection{In vivo imaging}

To demonstrate the in vivo imaging capability of the probe, dual-modality imaging of a mouse ear was conducted. All experimental animal procedures were carried out in conformity with the laboratory animal protocol approved by Laboratory Animal Care Committee at Shanghai Jiao Tong University. Before the experiment, the mouse was anesthetized with pentobarbital and positioned on an animal platform. Figure 7A shows the experimental setup. A water tank with a window opened at the bottom was used. The window was further sealed by a transparent and thin plastic wrap, which allowed transmission of the laser beam/white light and photoacoustic waves. Ultrasound gel was applied between the mouse ear and the plastic wrap to facilitate acoustic coupling. A $\mathrm{Z}$ stage was used to adjust the mouse to a proper height. As described previously, WLM mode was employed to facilitate the focus alignment as well as identify a region of interest, which showcases the ability of image guidance in practical applications. A region with adjacent arteries and veins was selected, as shown in Figure 7B. As indicated in Figure 7B, the vessels with different diameters can be clearly identified in WLM mode, and thus, arteries (corresponding to smaller diameter) and veins (corresponding to larger diameter) can be easily distinguished $[5,12]$. Then, PAM with the excitation wavelength of $532 \mathrm{~nm}$ was performed by linear scanning with scanning step size of $5 \mu \mathrm{m}$. The pulsed laser energy used was $\sim 305 \mathrm{~nJ}$, and the focus was aligned slightly below the tissue surface to ensure that the laser fluence was below the American National Standards Institute (ANSI) safety limit for skin surface $\left(20 \mathrm{~mJ} / \mathrm{cm}^{2}\right.$ for the visible spectral range). Figure $7 \mathrm{C}$ shows the XY MAP photoacoustic image of the vasculature in the dashed box region in Figure 7B. As expected, the PAM image presents a similar pattern to 
(A)

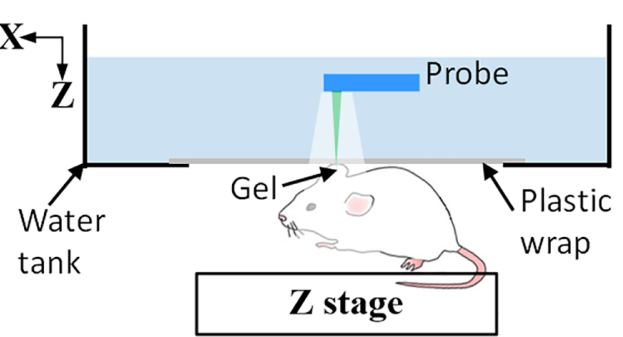

(B)

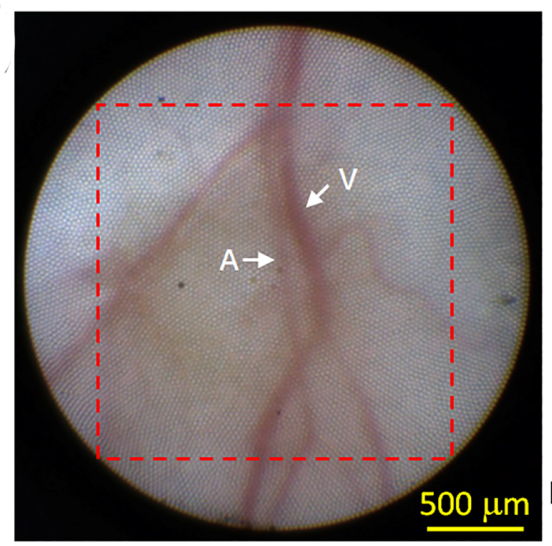

(C)

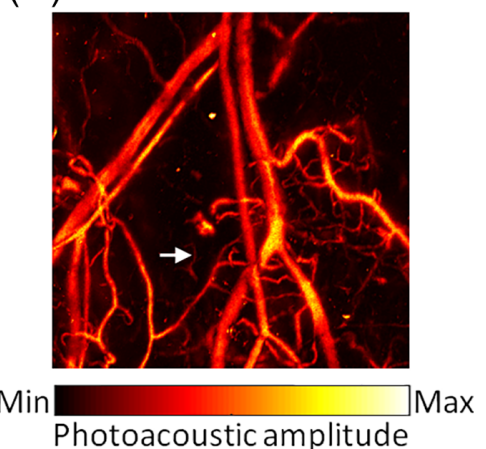

F I G URE 7 A, Schematic of experimental setup. White-light microscopy (WLM) image (B) and XY maximum amplitude projection (MAP) photoacoustic image (C) of the mouse ear in vivo. $\mathrm{B}, \mathrm{C}$, The same scale bar. A, artery; $\mathrm{V}$, vein the WLM image. Moreover, compared with the WLM image, the PAM image is able to reveal much finer structures down to $<8 \mu \mathrm{m}$, as indicated by the arrow in Figure 7C, by virtue of the high resolution and high contrast of PAM. The results show that WLM mode is advantageous for quick image guidance, and PAM mode is complementary to delineate detailed features of vasculature.

\section{4 | DISCUSSION}

The novelty of this work lies in the unique probe design capable of OR-PAM and WLM as well as much miniaturized probe diameter of $1.7 \mathrm{~mm}$ and high resolution of $7.2 \mu \mathrm{m}$ (OR-PAM), which paves the road toward an endoscope to facilitate clinical PAE applications. Compared with existing works [24,33], our probe has superior advantages in terms of miniature size and high resolution.

Our probe and system have several aspects to improve imaging capability and to facilitate clinical applications. First, the current arrangement of the acoustic transducer to align acoustic detection with laser excitation may result in limited imaging depth range. To realize larger imaging depth range, a design by using a gold-coated thin film for better overlap of laser excitation and acoustic detection regions would be taken into account in future [28]. Second, OR-PAM at excitation wavelengths of 532 and $680 \mathrm{~nm}$ was demonstrated. As a matter of fact, the imaging fiber bundle is able to provide illumination with diverging laser beams by utilizing multiple cores of the imaging fiber bundle [8]. Therefore, AR-PAM mode can be further developed using the broad illumination, which will lead to deeper penetration by utilizing diffused light at the expense of lateral resolution. Third, two simple scanning schemes were demonstrated in this work for convenience, which may not be applicable to clinical PAE applications. The prototype should be further developed to become a practical endoscope by integrating scanning capability so as to facilitate clinical PAE applications. One could consider integrating a torque coil to realize rotational scanning $[24,31,34]$. In this scheme, multiple MMFs can be used to deliver the white-light source for WLE mode [39]. Alternatively, raster scanning can be realized by utilizing the imaging fiber bundle $[6,32]$. In this scheme, a miniature ultrasonic transducer with wide-angle and high-sensitivity ultrasound detection has to be employed [32, 47]. The details are elaborated in Supporting Information. It is worth noting that the above approaches to realize scanning capability are expected to still keep the novelty demonstrated in the prototype.

The linear scanning speed of our current system is mainly limited by the PRF of $1 \mathrm{kHz}$ of the laser and the response time of the $2 \mathrm{D}$ motorized stage, which leads to the scanning time of $<4$ min (for $200 \times 200$ pixels with scanning step size of $5 \mu \mathrm{m}$ ), as mentioned previously. That is, the B-scan rate is $>0.83 \mathrm{~Hz}$. To further improve the imaging speed, a laser with higher PRF (up to $800 \mathrm{kHz}$ available [48]) and motors with faster response time can be employed. As for the rotational scanning (by a torque coil) and raster scanning (by the imaging fiber bundle) mentioned above, their scanning speed is discussed as follows. For the former, a motor can be used to realize 
rotational scanning of the torque coil [24, 31], while for the latter, a galvanometer mirror can be adopted to realize fast raster scanning. Thus, for the two scanning approaches, the scanning speed will be restricted by the PRF of a laser and the speed of the integrated scanner. According to previous studies [24, 31, 48], the B-scan rate of 5 and $100 \mathrm{~Hz}$ for the rotational scanning and raster scanning, respectively, should be technically possible. High imaging speed would facilitate clinical PAE applications, such as minimizing the issue of peristalsis of the gastrointestinal tract during PAE image acquisition.

In this study, the laser energy used for tissue imaging (Figures 4 and 7) was $\sim 305$ and $\sim 750 \mathrm{~nJ}$ (per pulse) for 532 and $680 \mathrm{~nm}$ lasers, respectively. The fluence at the focus can thus be calculated as $\sim 0.75 \mathrm{~J} / \mathrm{cm}^{2}$ $(532 \mathrm{~nm})$ and $\sim 0.64 \mathrm{~J} / \mathrm{cm}^{2}(680 \mathrm{~nm})$, which is comparable to that used in other OR-PAM studies [5, 7, 9, 13, 14]. In addition, as mentioned previously, the laser was focused below the tissue surface to conform to the ANSI safety limit.

\section{5 | CONCLUSIONS}

In summary, a 1.7-mm-diameter dual-modality probe capable of PAM and WLM was developed. High resolution of 7.2 and $31.3 \mu \mathrm{m}$ was achieved for PAM and WLM modes, respectively. Since PAM and WLM modes share the same optical beam path for light focusing (for PAM) and image formation (for WLM), their focal planes coincide with each other, which facilitates the quick focus alignment for PAM mode. Further, WLM mode also provides complementary information and can be used for image guidance in practical applications. By exploiting 532 and $680 \mathrm{~nm}$ lasers for PAM mode, we demonstrated scalable lateral resolution and imaging depth, and the potential for spectroscopic imaging to obtain functional information. Functional imaging such as blood oxygen saturation $\left(\mathrm{sO}_{2}\right)$ and blood oxygen metabolic rate would provide invaluable information for accurate diagnosis of diseases. To reveal $\mathrm{sO}_{2}$ by the probe, upgrading our laser system to have another excitation wavelength with high absorption of blood (eg, $\sim 560 \mathrm{~nm}$ ) is required [5, 10, 15]. High-quality dual-modality images were demonstrated in phantom and in vivo animal studies. The probe has advantages of dual modality, miniature size and high resolution, which has potential to open up new opportunities for PAE in biomedical applications.

\section{ACKNOWLEDGMENT}

This work was supported by National Natural Science Foundation of China (NSFC) (61775134).

\section{CONFLICT OF INTEREST}

The authors declare no financial or commercial conflict of interest.

\section{AUTHOR BIOGRAPHIES}

Please see Supporting Information online.

\section{ORCID}

Sung-Liang Chen (D) https://orcid.org/0000-0002-0572-

5110

\section{REFERENCES}

[1] P. Beard, Interface Focus 2011, 1(4), 602.

[2] L. V. Wang, S. Hu, Science 2012, 335(6075), 1458.

[3] J. Yao, L. V. Wang, Laser Photon. Rev. 2013, 7(5), 758.

[4] K. Maslov, H. F. Zhang, S. Hu, L. V. Wang, Opt. Lett. 2008, 33 (9), 929.

[5] S. Hu, K. Maslov, L. V. Wang, Opt. Lett. 2011, 36(7), 1134.

[6] P. Hajireza, W. Shi, R. J. Zemp, Opt. Lett. 2011, 36(20), 4107.

[7] J. Yao, C. H. Huang, L. Wang, J. M. Yang, L. Gao, K. I. Maslov, J. Zou, L. V. Wang, J. Biomed. Opt. 2012, 17(8), 080505.

[8] W. Xing, L. Wang, K. Maslov, L. V. Wang, Opt. Lett. 2013, 38 (1), 52.

[9] J. Chen, R. Lin, H. Wang, J. Meng, H. Zheng, L. Song, Opt. Express 2013, 21(6), 7316.

[10] L. Wang, K. Maslov, L. V. Wang, Proc. Natl. Acad. Sci. U. S. A. 2013, 110(15), 5759.

[11] P. Hai, J. Yao, K. I. Maslov, Y. Zhou, L. V. Wang, Opt. Lett. 2014, 39(17), 5192.

[12] B. Ning, M. J. Kennedy, A. J. Dixon, N. Sun, R. Cao, B. T. Soetikno, R. Chen, Q. Zhou, K. K. Shung, J. A. Hossack, S. Hu, Opt. Lett. 2015, 40(6), 910.

[13] G. Wissmeyer, D. Soliman, R. Shnaiderman, A. Rosenthal, V. Ntziachristos, Opt. Lett. 2016, 41(9), 1953.

[14] T. J. Allen, J. Spurrell, M. O. Berendt, O. Ogunlade, S. U. Alam, E. Z. Zhang, D. J. Richardson, P. C. Beard, J. Biomed. Opt. 2018, 23(12), 126502.

[15] C. Liu, Y. Liang, L. Wang, Biomed. Opt. Express 2019, 10(6), 3061.

[16] H. F. Zhang, K. Maslov, G. Stoica, L. V. Wang, Nat. Biotechnol. 2006, 24(7), 848.

[17] V. Periyasamy, N. Das, A. Sharma, M. Pramanik, J. Biophoton. 2019, 12(5), e201800357.

[18] J. M. Yang, K. Maslov, H. C. Yang, Q. Zhou, K. K. Shung, L. V. Wang, Opt. Lett. 2009, 34(10), 1591.

[19] J. M. Yang, R. Chen, C. Favazza, J. Yao, C. Li, Z. Hu, Q. Zhou, K. K. Shung, L. V. Wang, Opt. Express 2012, 20(21), 23944.

[20] J. M. Yang, C. Favazza, R. Chen, J. Yao, X. Cai, K. Maslov, Q. Zhou, K. K. Shung, Nat. Med. 2012, 18(8), 1297.

[21] S. L. Chen, Z. Xie, T. Ling, L. J. Guo, X. Wei, X. Wang, Opt. Lett. 2012, 37(20), 4263.

[22] K. Jansen, G. van Soset, A. F. van der Steen, Ultrasound Med. Biol. 2014, 40(6), 1037.

[23] X. Bai, X. Gong, W. Hau, R. Lin, J. Zheng, C. Liu, C. Zeng, X. Zou, H. Zheng, L. Song, PLoS One 2014, 9(3), e92463.

[24] J. M. Yang, C. Li, R. Chen, Q. Zhou, K. K. Shung, L. V. Wang, J. Biomed. Opt. 2014, 19(6), 066001. 
[25] H. Guo, C. Song, H. Xie, L. Xi, Opt. Lett. 2017, 42(22), 4615.

[26] D. Jin, F. Yang, Z. Chen, S. Yang, D. Xing, Appl. Phys. Lett. 2017, 111(10), 103703.

[27] D. Cai, G. Li, D. Xia, Z. Li, Z. Guo, S. L. Chen, Opt. Express 2017, 25(17), 20162.

[28] X. Dai, H. Yang, T. Shan, H. Xie, S. A. Berceli, H. Jiang, ACS Photon. 2017, 4(1), 174.

[29] K. Xiong, S. Yang, X. Li, D. Xing, Opt. Lett. 2018, 43(8), 1846.

[30] Y. Qu, C. Li, J. Shi, R. Chen, S. Xu, H. Rafsanjani, K. Maslov, H. Krigman, L. Garvey, P. Hu, P. Zhao, K. Meyers, E. Diveley, S. Pizzella, L. Muench, N. Punyamurthy, N. Goldstein, O. Onwumere, M. Alisio, K. Meyenburg, J. Maynard, K. Helm, J. Slaughter, S. Barber, T. Burger, C. Kramer, J. Chubiz, M. Anderson, R. McCarthy, S. K. England, G. A. Macones, Q. Zhou, K. K. Shung, J. Zou, M. J. Stout, M. Tuuli, L. V. Wang, J. Biomed. Opt. 2018, 23(12), 121617.

[31] Y. Li, R. Lin, C. Liu, J. Chen, H. Liu, R. Zheng, X. Gong, L. Song, J. Biophoton. 2018, 11(10), e201800034.

[32] G. Li, Z. Guo, S. L. Chen, IEEE Sens. J. 2019, 19(3), 909.

[33] R. Ansari, N. Huynh, E. Zhang, A. E. Desjardins, P. C. Beard, Proc. SPIE 2019, 10878, 1087804.

[34] Y. Li, G. Lu, J. J. Chen, J. C. Jing, T. Huo, R. Chen, L. Jiang, Q. Zhou, Z. Chen, Photoacoustics 2019, 15, 100138.

[35] M. Basij, Y. Yan, S. S. Alshahrani, H. Helmi, T. K. Burton, J. W. Burmeister, M. M. Dominello, I. S. Winer, M. Mehrmohammadi, Photoacoustics 2019, 15, 100139.

[36] J. Sun, C. Shu, B. Appiah, R. Drezek, J. Biomed. Opt. 2010, 15 (4), 040502.

[37] M. Hughes, T. P. Chang, G. Z. Yang, Biomed. Opt. Express 2013, 4(12), 2781.

[38] L. C. Chen, K. Yoshimura, S. Ninomiya, S. Takeda, K. Hiraoka, Mass Spec. 2017, 6(3), S0070.

[39] A. Burkhardt, J. Walther, P. Cimalla, M. Mehner, E. Koch, J. Biomed. Opt. 2012, 17(7), 071302.

[40] L. Kirsten, M. Schindler, J. Morgenstern, M. T. Erkkilä, J. Golde, J. Walther, P. Rottmann, M. Kemper, M. Bornitz,
M. Neudert, T. Zahnert, E. Koch, J. Biomed. Opt. 2018, 24(3), 031017.

[41] P. S. Thong, M. Olivo, W. W. Chin, R. Bhuvaneswari, K. Mancer, K. C. Soo, Br. J. Cancer 2019, 101(9), 1580.

[42] J. Glatz, J. Varga, P. B. Garcia-Allende, M. Koch, F. R. Greten, V. Ntziachristos, J. Biomed. Opt. 2013, 18(10), 101302.

[43] T. Thomas, D. Gilbert, P. V. Kaye, I. Penman, G. P. Aithal, K. Ragunath, Surg. Endosc. 2010, 24(5), 1110.

[44] T. Ling, S. L. Chen, L. J. Guo, Appl. Phys. Lett. 2011, 98(20), 204103.

[45] M. Kyrish, R. Kester, R. Richards-Kortum, T. Tkaczyk, Proc. SPIE 2010, 7558, 755807.

[46] K. Vyas, M. Hughes, B. G. Rosa, G. Z. Yang, Biomed. Opt. Express 2018, 9(10), 4649.

[47] J. A. Guggenheim, J. Li, T. J. Allen, R. J. Colchester, S. Noimark, O. Ogunlade, I. P. Parkin, I. Papakonstantinou, A. E. Desjardins, E. Z. Zhang, P. C. Beard, Nature Photon. 2017, 11, 714.

[48] J. Kim, J. Y. Kim, S. Jeon, J. W. Baik, S. H. Cho, C. Kim, Light Sci. Appl. 2019, 8, 103.

\section{SUPPORTING INFORMATION}

Additional supporting information may be found online in the Supporting Information section at the end of this article.

How to cite this article: Li G, Ye Z, Liang S, Chen S-L. Miniature probe for dual-modality photoacoustic microscopy and white-light microscopy for image guidance: A prototype toward an endoscope. J. Biophotonics. 2020;13: e201960200. https://doi.org/10.1002/jbio.201960200 